\title{
ANÁLISE DA EVOLUÇÃO TEMPORAL DA MORBIDADE HOSPITALAR DE IDOSOS NA REGIÃO DA AMUSEP ENTRE OS ANOS DE 2000 - 2010*
}

\author{
Ana Paula Serra de Araújo \\ Raquel Cristina Luis Mincoff \\ Priscilla Hellen Martinez Blanco \\ Tarcisio Vitor Augusto Lordani \\ Érica Simionato Machado \\ Patricia Domingos Noro da Silva \\ Daniel Vicentini de Oliveira
}

Mestres em Promoção da Saúde pelo Centro Universitário Cesumar - UNICESUMAR, Maringá (PR), Brasil.

\section{Mirian Ueda Yamaguchi}

Farmacêutica; Doutora em Ciências Farmacêuticas pela Universidade Estadual de Maringá - UEM; Docente no curso de Mestrado em Promoção da Saúde do Centro Universitário Cesumar - UNICESUMAR, Maringá (PR), Brasil.

E-mail: anasaraujo@hotmail.com

\footnotetext{
* Artigo desenvolvido durante a disciplina intitulada Atividade Interdisciplinar I do Mestrado em Promoção da Saúde pelo Centro Universitário Cesumar
}

RESUMO: O presente estudo tem como objetivo analisar comparativamente a evolução da morbidade hospitalar de idosos na região da Associação dos Municípios do Setentrião Paranaense (AMUSEP), entre os anos de 2000 e 2010, com base nos dados do Sistema de Informações Hospitalares do Sistema Único de Saúde (SIHSUS). Os grupos foram analisados de acordo com as causas constantes na $10^{a}$ revisão da Classificação Internacional da Doença e Problemas Relacionados à Saúde (CID-10) disponibilizados no DATASUS. Os resultados mostraram declínio nas taxas totais de morbidade referentes às doenças do sistema nervoso, aparelho circulatório, respiratório, causas mal definidas e causas externas, com taxas respectivas de $17,46 \%$, $69,10 \%, 4,52 \%, 57,26 \%$ e $1,81 \%$ no ano 2000 e em 2010 passaram respectivamente para $5,87 \%, 39,14 \%, 36,48 \%, 2,81 \%$ e $0,97 \%$. Em contrapartida, houve aumento das taxas de morbidade por doenças infecciosas e parasitárias, neoplasias, doenças do sangue, órgãos hematopoéticos e transtornos imunitários, aparelho geniturinário, lesões, envenenamento e algumas consequências externas, que no ano de 2000 representaram taxas anuais de 7,51\%, 4,25\%, 7,08\%, 7,67\%, e 7,91\% e em 2010 passaram a apresentar taxa de $12,21 \%, 27,61 \%$, $9,15 \%, 8,49 \%$ e $10,97 \%$, respectivamente. A realização deste estudo possibilitou não apenas o conhecimento das tendências de internações por morbidade de idosos residentes na região da AMUSEP, como também a constatação da necessidade de implantação de políticas públicas e programas de assistência à saúde capazes de reduzir essas taxas e modificar o perfil da saúde da população.

PALAVRAS-CHAVE: AMUSEP; CID-10; Idoso; Morbidade.

\section{EVOLUTION OF HOSPITAL MORBIDITY OF ELDERLY POPULATION WITHIN THE AMUSEP AREA, BET WEEN 2000 AND 2010}

ABSTRACT: The evolution of hospital morbidity in the elderly population within the area of the Association of Municipalities of the North of Paraná (AMUSEP), between 2000 and 2012, is comparatively analyzed from data retrieved from the Hospital Information System of the Brazilian Health System (SIH-SUS). Groups were analyzed according to causes in the International Classification of Diseases and Related Health Problems (CID-10) available on DATASUS. Results showed a decrease in total morbidity rates for diseases of the nervous system, circulatory and respiratory apparatus, badly defined causes and external causes, with respective rates $17.46 \%, 69.10 \%, 4.52 \%, 57.26 \%$ and $1.81 \%$ for 2000 , and $5.87 \%, 39.14 \%, 36.48 \%, 2.81 \%$ and $0.97 \%$ for 2010 . On the other hand, there was a yearly morbidity increase rate in infectious and parasite-caused diseases, cancer, blood diseases, hematopoietic organs and immune disorders, genitourinary apparatus, 
lesions, poisoning and several external consequences with $7.51 \%, 4.25 \%, 7.08 \%, 7.67 \%$ and $7.91 \%$, respectively, in 2000 , and with $12.21 \%, 27.61 \%, 9.15 \%, 8.49 \%$ and $10.97 \%$, respectively, in 2010 . Current analysis made possible not merely knowledge on hospitalization trends for morbidity in the elderly of the AMUSP area but also the need to establish health public policies and assistance programs to reduce rates and change the population's health profile

KEY WORDS: AMUSEP; CID-10; Elderly; Morbidity.

\section{INTRODUÇÃO}

A saúde é garantida a todo indivíduo através da constituição brasileira e sua manutenção envolve ações simultâneas dos Governos Municipal, Estadual e Federal. Para que a saúde seja preservada, devemos observar e compreender as mudanças que têm ocorrido no país no perfil de mortalidade e morbidade. $O$ acentuado envelhecimento populacional é fato decorrente da redução da fecundidade e do número de filhos, redução das taxas de mortalidade infantil e da mudança comportamental pela busca de uma melhor qualidade de vida. Diante destas mudanças, observa-se que cada vez há mais predominância de doenças crônico-degenerativas e de suas sequelas, resultando na procura cada vez maior pelos serviços de saúde e pela necessidade de ações comprometidas com a promoção e qualidade de saúde da população (SOUZA; SCOCHI; MARASCHIN, 2011).

Neste contexto, a análise das estatísticas de morbidade disponibilizadas em sistemas de informações em saúde do país, torna-se uma tarefa importante na avaliação do estado de saúde desta população e consequentemente uma estratégia de relevada importância para a geração de subsídios que possam ser utilizados no planejamento de ações de promoção da saúde (SANCHES et al., 2004). Além de ser uma ação de fundamental importância para todo e qualquer profissional da saúde, em especial aqueles que lidam diretamente como a prestação de serviços de assistências em saúde como é o caso dos profissionais médicos, enfermeiros, nutricionistas, fisioterapeutas, farmacêuticos, entre outros.

No Brasil, sistemas de informações em saúde têm representado fontes importantes para o conhecimento, planejamento e avaliação do sistema de saúde, por meio da realização de pesquisas científicas de seus dados (SANCHES et al., 2004).

A morbidade é um indicador de saúde muito importante, sendo definida como um termo genérico usado para designar o conjunto de casos de uma determinada afecção ou a soma de agravos à saúde que atingem um grupo de indivíduos (MATHIAS; JORGE; ANDRADE, 2006). Descrever o perfil da morbidade da população idosa é uma tarefa necessária para subsidiar ações que sejam adequadamente implantadas na região pesquisada, a fim de atender às maiores necessidades de saúde destes indivíduos (MATHIAS; JORGE; ANDRADE, 2006). Para que haja desfecho positivo em cada região estudada, é importante que as condições de saúde e das doenças sejam devidamente pesquisadas.

Os três principais sistemas de informações de morbidade do país referem-se ao Sistema de Informações Hospitalares do Sistema Único de Saúde (SIH/SUS), Sistema de Informações Ambulatoriais (SIA/SUS) e Sistema de Informação de Agravos de Notificação (SINAN). Porém, diferentemente do que ocorre com as informações sobre mortalidade, são escassos estudos com dados sobre a morbidade (LAURENTI; JORGE; GOTLIEB, 2004).

De acordo com Sanches et al. (2004) o SIH-SUS, desde sua implantação, representa um banco de dados de caráter administrativo, baseado na Autorização de Internação Hospitalar (AIH), que envolve um conjunto de dados referentes à identificação do paciente e à internação do mesmo. O SIH-SUS, por meio dos dados obtidos pela AIH, possibilita o conhecimento da morbidade hospitalar de determinada localidade, o que ajuda os gestores e profissionais da saúde nos controles de saúde e prevenção primária (BRASIL, 2007).

Para os dados de morbidade, além daqueles advindos das doenças de notificação obrigatória, das doenças e acidentes de trabalho, existem as estatísticas sobre saídas hospitalares, que incluem os diagnósticos e estão cadastrados no SIH-SUS (SANCHES et al., 2004; BRASIL, 2009).

Para melhor entendimento deste processo, vale salientar que nos Estados Unidos da América (EUA), a grande demanda por estatísticas de morbidade cresceu e culminou na aprovação, em 1995, de uma ata - Health 
Survey Act, que desde então produz estatísticas de morbidade e incapacidades, em nível nacional (SANCHES et al., 2004).

A necessidade de dados de doenças ou diagnósticos concretos para a população aumentou em grande escala, assim como a preocupação com os efeitos de problemas ambientais sobre a saúde. A poluição ambiental, as radiações ionizantes, a contaminação química e os efeitos sobre a saúde levaram à necessidade de estudos quantitativos e, dessa maneira, houve aumento do número de estudos epidemiológicos, particularmente a partir da segunda metade do século XX (SANCHES et al., 2004).

Atualmente, existe maior disponibilidade de dados referentes à morbidade hospitalar, que refletem, em parte, a ocorrência das formas clínicas mais severas das doenças. Quanto aos dados de morbidade de base não hospitalar, existe maior disponibilidade para as Doenças Infecciosas e Parasitárias (DIP), em especial para as doenças de notificação obrigatória (CARMO; BARRETO; SILVA-JUNIOR, 2003). Já a análise da morbidade por estas causas vem exigindo criações de grandes bancos de dados, especificamente desenhados para essa finalidade (MELLO; KOIZUMI, 2004).

O presente estudo objetivou analisar a evolução temporal da morbidade hospitalar de idosos (idade $\geq 60$ anos) na região da Associação dos Municípios do Setentrião Paranaense (AMUSEP), entre os anos de 2000 e 2010, com base nos dados do Sistema de Informações Hospitalares do Sistema Único de Saúde (SIH-SUS), conforme os grupos de causas constantes na $10^{\mathrm{a}}$ revisão da Classificação Internacional da Doença e Problemas Relacionados à Saúde (CID-10).

\section{METODOLOGIA}

Estudo descritivo e exploratório, com coleta de dados secundários de mortalidade hospitalar do SUS do Brasil, disponibilizados no SIH-SUS, do banco de dados do Sistema Único de Saúde (DATASUS) de domínio público e acesso irrestrito por intermédio da Internet.

Os municípios pesquisados integram os 30 municípios que compõem o Consórcio Intermunicipal de Saúde da Associação dos Municípios do Setentrião Paranaense (CISAMUSEP) sendo eles: Ângulo, Astorga, Atalaia, Colorado, Floraí, Floresta, Iguaraçu, Ivatuba, Lobato, Doutor Camargo, Flórida, Itaguajé, Itambé, Mandaguaçu, Mandaguari, Marialva, Maringá, Munhoz de Mello, Nossa Senhora das Graças, Nova Esperança, Ourizona, Paiçandu, Paranacity, Presidente Castelo Branco, Santa Fé, Santa Inês, Santo Inácio, São Jorge do Ivaí, Sarandi e Uniflor, que juntos possuem um contingente populacional de aproximadamente $700 \mathrm{mil}$ habitantes, em uma área de $6.997 \mathrm{~km}^{2}$, e que compõem o Consórcio Intermunicipal de Saúde da AMUSEP, denominado CISAMUSEP.

O universo do estudo foi constituído pelo número total de morbidades dos registros de internações no SUS de indivíduos com idade superior a 60 anos, por local de internação, no período de janeiro de 2000 a dezembro de 2010, informados no SIH-SUS; e as estimativas da população residente nos municípios da AMUSEP para o grupo etário de idosos, do Instituto Brasileiro de Geografia e Estatística (IBGE).

Para a avaliação da tendência da morbidade, foram considerados todos os capítulos da Classificação Internacional da Doença e Problemas Relacionados à Saúde (CID-10) apresentados no Quadro 1.

As informações de morbidade foram categorizadas segundo a CID-10, calculando-se a taxa de mortalidade por causa básica do óbito em referência ao total de idosos, residentes na região AMUSEP conforme o ano pesquisado, em razão do ano dos óbitos, para 1.000 habitantes segundo a fórmula do Coeficiente de Morbidade por Causas (CMC) apresentada abaixo:

$\mathbf{C M C}=\underline{\text { Número de casos por causa, tempo, lugar }} \times 1000$ População exposta

Posteriormente os dados obtidos foram transformados em gráficos e submetidos a análise estatística descritiva com o intuito de se verificar a curva de morbidade (ascendente ou descendente) na região AMUSEP, entre os anos de 2000 e 2010, e os municípios com maiores taxas de morbidade conforme a causa básica de internação segundo os capítulos da CID-10, e assim buscar correlações entre estes dados, e as possíveis explicações para a evolução da morbidade na região, e o 
que poderia vir a ser feito para melhorar a qualidade de vida da população atendida pelo CISAMUSEP.

Quadro 1. Capítulos da Classificação Internacional da Doença e Problemas Relacionados à Saúde (CID-10), disponibilizados no site do DATASUS, segundo seu código, número do capítulo e especificações

\begin{tabular}{|c|c|c|}
\hline CAPÍTULO & CID - 10 & CÓDIGO \\
\hline I & $\begin{array}{l}\text { Algumas doenças infecciosas e } \\
\text { parasitárias }\end{array}$ & A00-B99 \\
\hline II & Neoplasias/Tumores & C00-D48 \\
\hline III & $\begin{array}{l}\text { Doenças das glândulas endócrinas, } \\
\text { nutricionais e metabólicas }\end{array}$ & D50-D89 \\
\hline IV & $\begin{array}{l}\text { Doenças do sangue, órgãos } \\
\text { hematopoéticos e transtornos } \\
\text { imunitários }\end{array}$ & E00-E90 \\
\hline $\mathbf{V}$ & $\begin{array}{l}\text { Transtornos mentais e } \\
\text { comportamentais }\end{array}$ & F00-F99 \\
\hline VI & Doenças do sistema nervoso & G00-G99 \\
\hline VII & Doenças do olho e anexos & $\mathrm{H} 00-\mathrm{H} 59$ \\
\hline VIII & $\begin{array}{l}\text { Doenças do ouvido e da apófise } \\
\text { mastóide }\end{array}$ & $\mathrm{H} 60-\mathrm{H} 95$ \\
\hline IX & Doenças do aparelho circulatório & I00-I99 \\
\hline $\mathbf{X}$ & Doenças do aparelho respiratório & J00-J99 \\
\hline XI & Doenças do aparelho digestório & K00-K93 \\
\hline XII & $\begin{array}{l}\text { Doenças da pele e do tecido } \\
\text { subcutâneo }\end{array}$ & L00-L99 \\
\hline XIII & $\begin{array}{l}\text { Doenças do sistema osteomuscular e } \\
\text { tecido conjuntivo }\end{array}$ & M00-M99 \\
\hline XIV & Doença aparelho geniturinário & N00-N99 \\
\hline $\mathbf{X V}$ & Gravidez, parto e puerpério & 000-099 \\
\hline XVI & $\begin{array}{l}\text { Algumas afecções originadas no } \\
\text { período perinatal }\end{array}$ & P00-P96 \\
\hline XVII & $\begin{array}{l}\text { Malformações congênitas, } \\
\text { deformidade e anomalias } \\
\text { cromossômicas }\end{array}$ & Q00-Q99 \\
\hline XVIII & $\begin{array}{l}\text { Sintomas, sinais e achados } \\
\text { anormalidades clínicas e laboratoriais }\end{array}$ & R00-R99 \\
\hline XIX & $\begin{array}{l}\text { Lesões, envenenamento e algumas } \\
\text { consequências externas }\end{array}$ & S00-T98 \\
\hline $\mathbf{X X}$ & $\begin{array}{l}\text { Causas externas de morbidade } \mathrm{e} \\
\text { mortalidade }\end{array}$ & V01-Y98 \\
\hline
\end{tabular}

\section{RESULTADOS}

Assim como está ocorrendo em todo o Brasil, na região da AMUSEP, a população idosa vem apresentando crescimento, como demonstrado na Tabela 1.

Tabela 1. População idosa ( $\geq 60$ anos) da região AMUSEP entre os anos de 2000-2010

\begin{tabular}{lc}
\hline POPULAÇÃO IDOSA NA REGIÃO DA AMUSEP \\
\hline ANO & NÚMERO DE IDOSOS \\
\hline 2000 & 58.014 \\
2001 & 58.985 \\
2002 & 59.743 \\
2003 & 60.554 \\
2004 & 61.358 \\
2005 & 63.166 \\
2006 & 64.105 \\
2007 & 80.015 \\
2008 & 81.981 \\
2009 & 85.745 \\
2010 & 89.483 \\
\hline
\end{tabular}

Fonte: IBGE (2010, p. 1).

Em onze anos, nesta região do Paraná, nota-se que a situação do perfil de morbidade se deteve com maior predominância nas patologias e complicações devidas às doenças relacionadas ao CID IX (doenças do aparelho circulatório) e o CID X (doenças do sistema respiratório), como demonstrado abaixo na Figura 1.

Além disto, os casos de internações por acometimentos pelos CID VIII (Doenças do ouvido e da apófise mastoide); CID XV (Gravidez, parto e puerpério); CID XVI (Doenças do período perinatal) e CID XX (Causas externas mal definidas) apresentaram baixo acometimento entre os idosos desta região (Figura 1).

Atenção deve ser dada ao se considerar que o quadro de morbidade apresentado na Figura 1 diz respeito somente às internações subsidiadas financeiramente pelo SUS com a AIH/SUS, não sendo neste estudo computadas as internações particulares e de convênios de saúde.

Quando se analisam as taxas de morbidade de 2000 e 2010, referentes às doenças enquadradas no CID10, dos municípios que compõem a região da AMUSEP, pode-se observar que houve redução das taxas de morbidade por doenças do aparelho circulatório (CID 
IX) e respiratório (CID X), que estavam entre as maiores causas de morbidade no ano de 2000; como também houve queda das taxas de morbidade por doenças do sistema nervoso (CID VI) e do aparelho digestivo (CID XI). Em contrapartida, verificou-se acréscimo da taxa de morbidade por doenças infecciosas e parasitárias (CID I); neoplasias (CID II); doenças metabólicas, endócrinas e nutricionais (CID IV); doenças do aparelho geniturinário (CID XIV) e por lesões por envenenamento e algumas consequências de causas externas (CID XX), conforme demonstrado na Figura 2.

Os resultados apresentados nas Figuras 3 e 4 permitem a verificação das causas de internações de

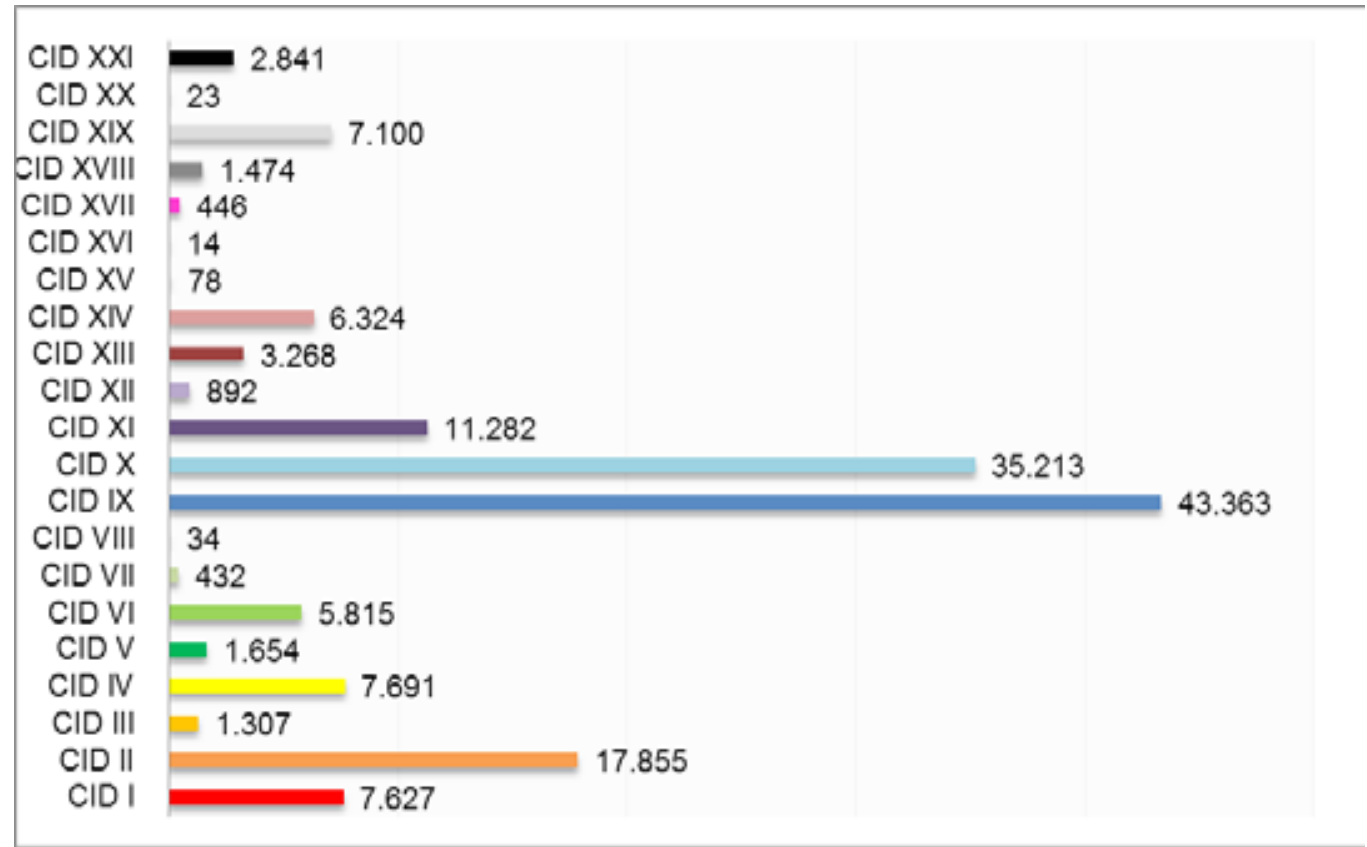

Figura 1. Número absoluto de internações de indivíduos com idade $\geq 60$ anos no SUS na região AMUSEP conforme causa básica de morbidade capítulos I a XXI da CID-10, no período de 2000 a 2010.

idosos por morbidades, demonstrando grande mudança no seu perfil comportamental durante o período de 2000-2010, no qual algumas doenças tiveram declínio na taxa de morbidade, como foi o caso das doenças do aparelho circulatório (CID 10 - IX); doenças do aparelho respiratório (CID 10 - X); causas externas de morbidade (CID 10 - XX). Outras patologias (Figura 3), como as doenças neoplásicas (CID 10 - II), aumentaram sua taxa de morbidade em $23,36 \%$ no período avaliado. 


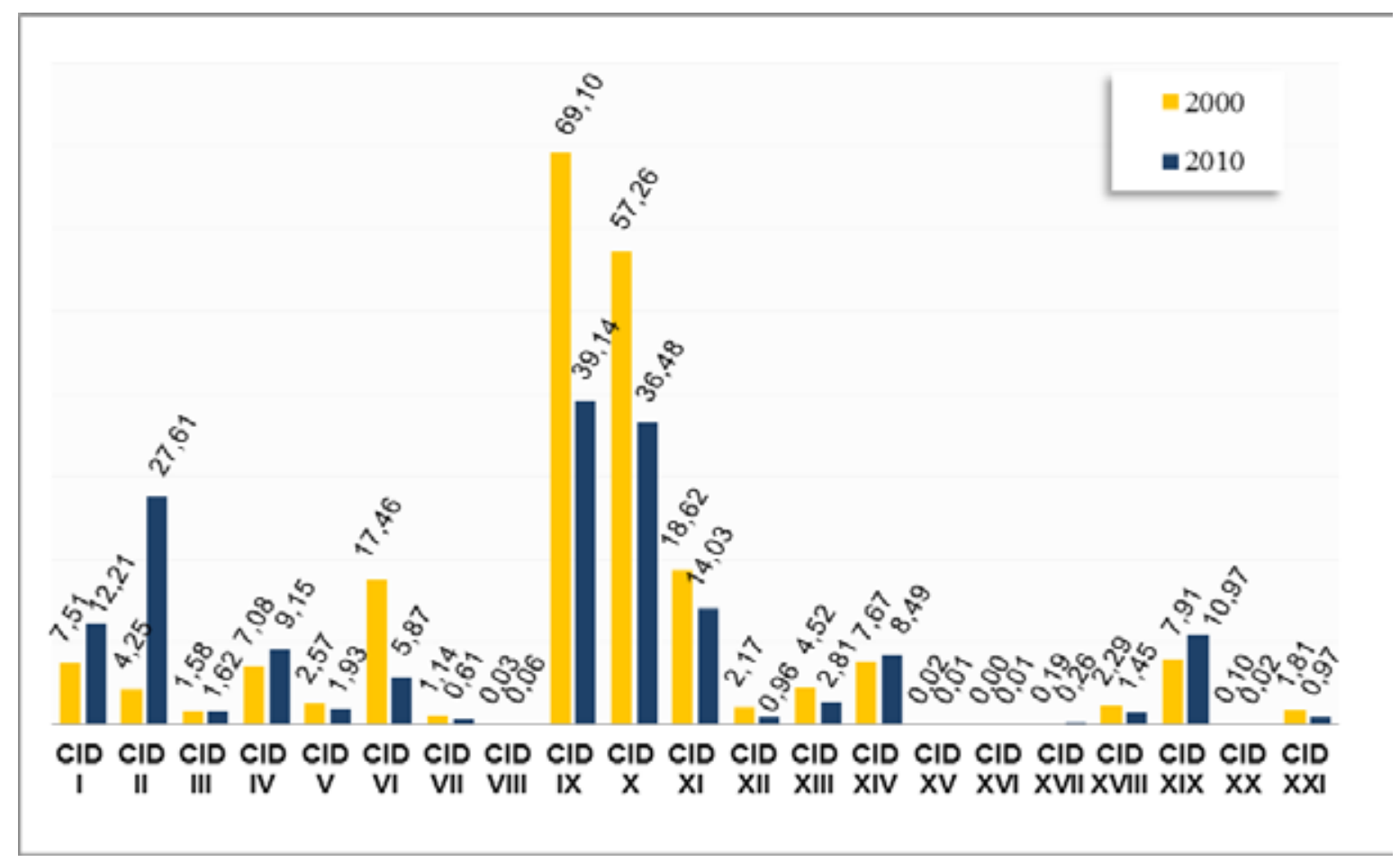

Figura 2. Taxas absolutas da morbidade de idosos ( $\geq 60$ anos) em internações do SUS, conforme causa básica constante nos capítulos da CID-10, na região da AMUSEP nos anos de 2000 e 2010.

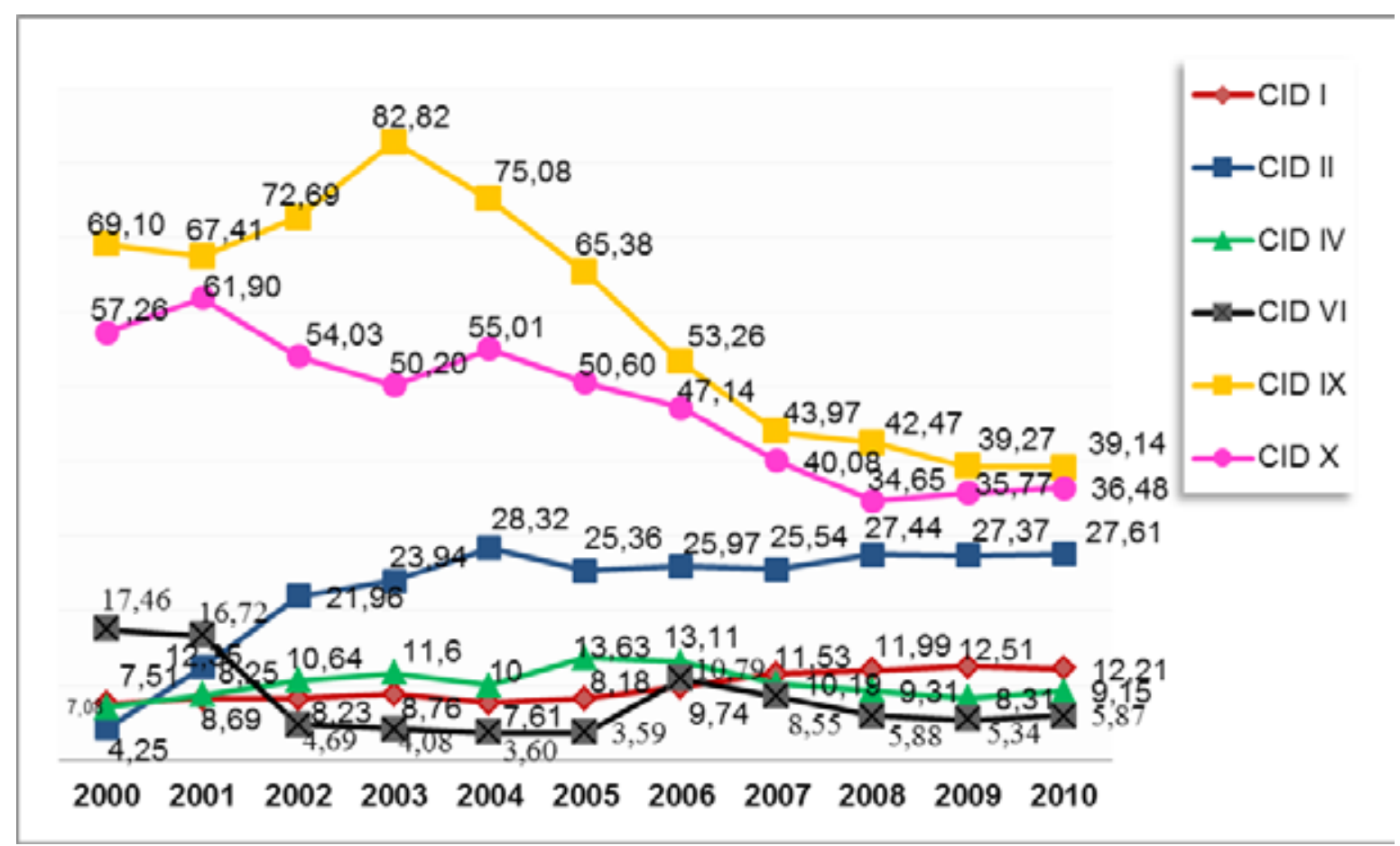

Figura 3. Evolução temporal da taxa de morbidade em internação do SUS de idosos ( $\geq 60$ anos), na região AMUSEP, no período de 2000 a 2010, conforme os capítulos CID 10 de I, II, IV, IX e X.

*Os capítulos da CID 10 - III, V, VI, VII, VIII, não constantes na figura, tiveram taxa de morbidade inferior a 1\%, na população idosa da AMUSEP no período pesquisado. 


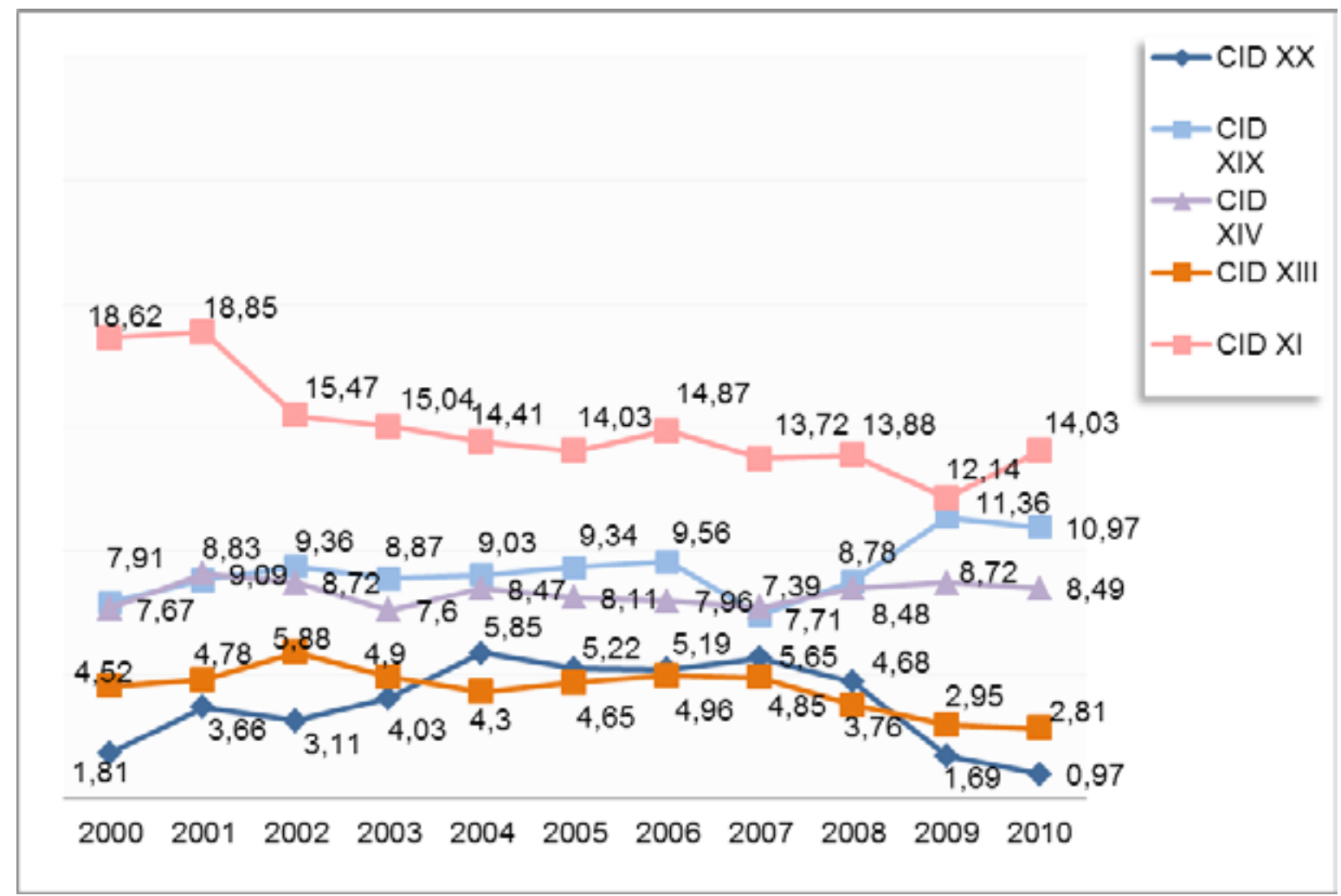

Figura 4. Evolução temporal da taxa de internação/morbidade, de idosos ( $\geq 60$ anos), por local de internação do SUS, na região AMUSEP, no período de 2000-2010, conforme os capítulos CID 10 de XI, XVIII, XIV, XIX e XXI.

*Os capítulos da CID 10; XI, XIII, XV, XVI, XVII, XVIII, não constantes na figura, tiveram taxa de morbidade inferior a 1\% na população idosa da AMUSEP no período pesquisado.

As causas de morbidade em internações de idosos no SUS na região AMUSEP, classificados nos capítulos CID 10, apresentaram-se bastante variáveis e em alguns períodos algumas destas causas obtiveram pico na sua taxa de prevalência, como no caso das internações por doenças do aparelho circulatório (CID 10 - IX) que no ano de 2003 se destaca na Figura 3, com taxa de morbidade na região de $82,82 \%$.

De acordo com os dados obtidos pelo estudo, entre os anos de 2000 e 2010 , houve modificação no padrão de morbidade de idosos na região da AMUSEP, referente às causas básicas de morbidade constantes na CID-10.

\section{DISCUSSÃO}

As doenças mais prevalentes em causa de morbidade entre os idosos são as denominadas de Doenças Crônicas Não Transmissíveis (DCNT). Entre essas, as principais são as doenças cardiovasculares, neoplásicas e as cerebrovasculares, cujo principal representante é o acidente vascular encefálico (AVE) (ANDRADE; RODRIGUES, 2002).

As patologias clínicas cujo fator de risco é a idade do indivíduo tendem a ter sua prevalência aumentada, conforme ocorre o envelhecimento populacional. Alguns exemplos disto podem ser verificados com a grande incidência e prevalência de patologias como a Doença de Alzheimer, diabetes mellitus, e alguns transtornos do sistema nervoso. Nasri (2008) cita que o risco de demência aumenta cerca de duas vezes mais a cada cinco anos, assim que o indivíduo completa 65 anos de idade, como também aumentam as queixas de doenças crônicas que induzem os idosos a ingerirem mais medicamentos $\mathrm{e}$ realizar exames de rotina.

As doenças dos aparelhos circulatório, respiratório e digestivo respondem por aproximadamente $60 \%$ das causas de internação em instituições conveniadas ao SUS, para ambos os sexos (LOYOLA-FILHO et al., 2004). A observação do predomínio de internações por doenças do aparelho circulatório e do aparelho respiratório entre idosos não se modifica do que foi 
observado anteriormente, no Brasil e nos EUA (HALL; OWINGS, 2000).

As principais causas de internação entre idosos correspondem às doenças cujo aparecimento e complicações podem ser reduzidas com a adoção individual de novos hábitos de vida, como por exemplo, redução do tabagismo e do consumo excessivo de álcool; dieta equilibrada, com baixo teor de gordura; prática de atividade física e por intervenções de medidas de promoção da saúde; atividades educativas; campanhas de vacinação contra gripe, atendimento domiciliar, entre outras doenças do aparelho circulatório, que representavam a primeira causa de morte entre os idosos brasileiros com mais de 60 anos de idade em 1980, 1991 e 1996, respectivamente (LIMA; COSTA; MAIA, 2000).

A redução nas taxas de morbidade por doenças do aparelho circulatório e do aparelho respiratório na região da AMUSEP nos anos pesquisados pode ser responsabilizada pela criação do Consórcio Intermunicipal de Saúde - CISAMUSEP em 2002, que atende atualmente cerca de 500 consultas/dia em várias especialidades médicas e odontológicas. Outra possível causa da redução das taxas de morbidade deve estar relacionada às medidas criadas pelo Ministério da Saúde, como o Sistema de Cadastramento e Acompanhamento de Hipertensos e Diabéticos (HIPERDIA) captados no Plano Nacional de Reorganização da Atenção à Hipertensão Arterial e ao Diabetes mellitus, em todas as unidades ambulatoriais do SUS. Tais medidas geram informações para os gerentes locais, gestores das secretarias municipais, estaduais e Ministério da Saúde e que garante medicação aos indivíduos hipertensos e diabéticos na rede básica de saúde (BUENO; MOTTA, 2011).

Segundo Ferrer et al. (2008), as doenças respiratórias em outros Estados do Brasil ocupam lugar de pouca relevância entre as doenças, entretanto na região Sul do Brasil, ocupa lugar de destaque e relaciona este achado aos quadros de gripe H1N1 (Influenza A H1N1) e a pneumonia.

O Brasil passou por transformações e a redução significativa das causas de morbidade e mortalidade por doenças parasitárias e infecciosas é reflexo dos investimentos em programas específicos de controle e prevenção, tais como campanhas de vacinação, ampliação de saneamento básico nos centros urbanos, melhoria nas condições dos locais de trabalho, aprovações de legislações sociais que apoiam os investimentos em políticas de habitação, saúde, transporte, entre outros (CHAIMOWICZ, 2007). Porém, ainda é possível se deparar na região da AMUSEP com situações onde a taxa de morbidade por doenças parasitárias e infecciosas se elevaram nos últimos anos.

As doenças parasitárias são responsáveis por considerável morbidade e mortalidade em todo o mundo e frequentemente estão presentes com sinais e sintomas não específicos, o que dificulta seu diagnóstico e sua abordagem terapêutica (MATOS; MURAI, 2007). Estas afecções à saúde da população possuem geralmente causas infecciosas e parasitárias. Sua incidência diminuiu nas regiões Norte, Nordeste e Centro-Oeste e mantiveram os níveis quase estáveis no Sul e Sudeste, não ultrapassando os 7,3\% em 2004 em todas as regiões (ANDRADE et al., 2010).

No estudo de Andrade et al. (2010), cujo objetivo foi investigar a prevalência de enteroparasitoses no grupo de idosos inscritos no Centro de Convivência da Terceira Idade, com ênfase nos fatores determinantes ambientais, verificou-se após o rastreamento coprológico de 51 idosos, infecção pelos protozoários Endolimax nana (52,0\%), Entamoeba coli (20,0\%), Giardia lamblia (20,0\%), e Entamoeba bistolytica (8,0\%). Entre os fatores ambientais relacionados à infecção dos idosos por esses protozoários, o déficit de rede de esgoto e a ingestão de alimentos crus em baixa proporção foram identificados, enquanto a bibliografia aponta a higiene pessoal e dos alimentos como os de maior relevância.

Louveison e Barros (2009), em seu estudo, relatam que em torno de $80 \%$ dos idosos apresentam pelo menos uma enfermidade crônica, sendo a hipertensão a mais prevalente, atingindo mais da metade deste contingente populacional e impactando na crescente demanda e utilização dos serviços de saúde, principalmente hospitalares. No presente estudo, na região da AMUSEP as causas de morbidade por doenças do aparelho circulatório apresentaram-se como a maior causa, seguida das doenças do aparelho respiratório.

$\mathrm{O}$ aumento das causas de morbidade por doenças 
neoplásicas em pessoas com idade $\geq 60$ anos na região da AMUSEP foi um achado semelhante ao de outro estudo que avaliou a tendência de mortalidade por doenças neoplásicas em 10 capitais brasileiras entre os anos de 1980 e 2000, e que verificou que no grupo etário acima de 60 anos, ocorreu um aumento de casos de neoplasias/ mortalidade por neoplasias de 126,6\% entre os homens e $133 \%$ entre as mulheres em comparação a grupos etários mais jovens (CERVI; HERMSDORFF; RIBEIRO, 2005).

Segundo Mathias, Jorge e Andrade (2006), as intervenções relacionadas ao envelhecimento devem ter por objetivo assegurar aos idosos o prolongamento da vida com manutenção da capacidade funcional, física, mental e de qualidade aceitável. É necessário que ações envolvam acessibilidade aos serviços, procurando atender aos idosos de maneira integral (social, ocupacional, moradia, seguridade, alimentação, transporte e recreação). Assim como são necessárias novas pesquisas sobre a temática, onde se contemplem novas informações que possam contribuir de modo mais fidedigno para a compreensão da evolução da situação de saúde da população, e para que possam ser desenvolvidas ações cujo objetivo não seja apenas promover saúde, mas aumentar o empoderamento dos profissionais de saúde sobre a temática em questão.

\section{CONSIDERAÇÕES FINAIS}

$\mathrm{O}$ presente estudo indicou que as maiores causas de morbidade de idosos na região da AMUSEP são as doenças relacionadas ao aparelho circulatório e respiratório, embora as taxas de morbidade destas doenças tenham sofrido redução nos últimos anos.

Verificou-se com clareza o aumento da taxa de morbidade de outras doenças que anteriormente estavam controladas, como foi o caso das doenças parasitárias e infecciosas que tiveram um aumento de $7 \%$. As doenças gastrointestinais e geniturinárias também apresentaram aumento da taxa de morbidade. Houve, portanto, redução na taxa das doenças mais agressivas, porém o aumento das patologias resultantes da mudança demográfica, hábitos de higiene e alimentares.

$\mathrm{O}$ conhecimento das causas mais comuns de morbidade na população idosa da AMUSEP permitiu a constatação da necessidade de implantação de políticas públicas e programas de assistência à saúde capazes de reduzir essas taxas e melhorar o perfil de saúde da população idosa.

\section{REFERÊNCIAS}

ANDRADE, E. C. et al. Parasitoses intestinais: uma revisão sobre seus aspectos sociais, epidemiológicos, clínicos e terapêuticos. Rev. APS, v. 13, n. 2, p. 231-240, abr.jun. 2010.

ANDRADE, O. G.; RODRIGUES, R. A. P. Abordagem holística do sistema de cuidado familiar do idoso com acidente vascular cerebral. Ciênc. Cuid. Saúde, v. 1, n. 1, p. 185-191, jan./jun. 2002.

BRASIL. Ministério da Saúde. A experiência brasileira em sistemas de informação em saúde. Brasília: Ministério da Saúde, 2009.

BRASIL. Ministério da Saúde. Secretaria de Atenção à Saúde. Departamento de Regulação, Avaliação e Controle. Manual técnico do sistema de informação hospitalar. Brasília: Ministério da Saúde, 2007.

BUENO,J. B. D.; MOTTA,J. D. Instrumentos para efetivação do direito a saúde como direito da personalidade: Consórcio público intermunicipal de saúde do setentrião paranaense - CISAMUSEP e sua aplicabilidade. Rev. Jurídica Cesumar - Mestrado, v. 11, n. 2, p. 481-494, jul./dez. 2011.

CARMO, E. H.; BARRETO, M. L.; SILVA-JUNIOR, J. B. Mudanças nos padrões de morbimortalidade da população brasileira: os desafios para um novo século. Epidemiol. Serv. Saúde, v. 12, n. 2, p. 63-75, jun. 2003.

CERVI, A.; HERMSDORFF, H. H. M.; RIBEIRO, R. C. L. Tendência da mortalidade por doenças neoplásicas em 10 capitais brasileiras de 1980 a 2000. Rev. Bras. Epidemiol., v. 8, n. 4, p. 407-418, dez. 2005.

CHAIMOWICZ, F. Saúde dos idosos brasileiros às vésperas do século XXI: problemas, projeções e alternativas. Rev. 
Saúde Pública, v. 31, n. 2, p. 184-200, abr. 2007.

FERRER, A. L. M.; MARCON, S. S.; SANTANA, R. G. Morbidade hospitalar em idosos antes e após a vacinação contra influenza no estado do Paraná. Rev. Lat-Am. Enfermagem, v. 16, n. 5, p. 832-837, set./out. 2008.

HALL, M. J.; OWINGS, M. F. National Hospital Discharge Survey: 2000 Annual Summary With Detailed Diagnosis and Procedure Data. Vital Healt Sta., v. 12, n. 153, p. 1-20, dec. 2000.

IBGE. Instituto Brasileiro de Geografia e Estatística. Atlas do Censo Demográfico 2010. 2010. Disponível em: http://www.ibge.gov.br/home/estatistica/populacao/ censo2010/default_atlas.shtm. Acesso em: 10 ago. 2012.

LAURENTI, R.; JORGE, M. H. P. M.; GOTLIEB, S. L. D. A confiabilidade dos dados de mortalidade e morbidade por doenças crônicas não transmissíveis. Ciên. Saúde Coletiva, v. 9, n. 4, p. 909-920, out./dez. 2004.

LIMA, M. F.; COSTA, H.; MAIA, R. Diagnóstico da Situação de Saúde da População Idosa Brasileira: um Estudo da Mortalidade e das Internações Hospitalares Públicas. Inf. Epidemiol. SUS, v. 9, n. 1, p. 23-41, jan./mar. 2000.

LOUVISON, M. C. P.; BARROS, S. Políticas públicas e envelhecimento: a construção de uma política de direitos e os desafios da atenção integral à saúde da pessoa idosa no SUS. Bol. Instituto Saúde, v. 2, n. 47, p. 1-11, abr. 2009.

LOYOLA-FILHO, A. I. et al. Causas de internações hospitalares entre idosos brasileiros no âmbito do Sistema Único de Saúde. Epidemiol. Serv. Saúde, v. 13, n. 4, p. 229-238, dez. 2004.

MATHIAS, T. A. F.; JORGE, M. H. P. M.; ANDRADE, O. G. Morbimortalidade por causas externas na população idosa residente em município da região Sul do Brasil. Rev. Lat-Am. Enfermagem, v. 14, n. 1, p. 17-24, jan./ fev. 2006.

MATOS, A. S.; MURAI, H. C. Prevalência de parasitoses intestinais por Helmintos e Protozoários em idosos. Rev. Enferm. UNISA, v. 1, n. 6, p. 9-14, jan. 2005.
MELLO, J. M. H. P.; KOIZUMI, M. S. Gastos governamentais do SUS com internações hospitalares por causas externas: análise no Estado de São Paulo. Rev. Bras. Epidemiol., v. 7 , n. 2, p. 228-238, jun. 2004.

NASRI, F. O envelhecimento populacional no Brasil. Einstein, v. 6, n. 1, p. 54-56, jun. 2008.

SANCHES, K. R. B. et al. Sistemas de informação em saúde. In: MEDRONHO, R. A. (Org). Epidemiologia. São Paulo: Atheneu, 2004. p. 337-357.

SOUZA, E. A.; SCOCHI, M. J.; MARASCHIN, M. S. Estudo da morbidade em uma população idosa. Esc. Anna Nery, v. 15, n. 2, p. 380-388, abr. jun. 2011.

Recebido em: 21 de janeiro de 2015 Aceito em: 08 de junbo de 2015 